\title{
Primary brewery wastewater as feedstock for the yeast Rhodosporidium toruloides and the microalga Tetradesmus obliquus mixed cultures with lipid production
}

\author{
Carla Dias $^{a}$, Alberto Reis ${ }^{a}$, José A.L. Santos ${ }^{\mathrm{b}, \mathrm{c}}$, Luisa Gouveia ${ }^{\mathrm{a}, \mathrm{d}}$, Teresa Lopes da Silva ${ }^{\mathrm{a}, *}$ \\ ${ }^{a}$ Laboratório Nacional de Energia e Geologia, I.P., Unidade de Bioenergia e Biorefinarias - UBB, Estrada do Paço do Lumiar 22, 1649-038, Lisboa, Portugal \\ ${ }^{\mathrm{b}}$ Departamento de Bioengenharia, Instituto Superior Técnico, Universidade de Lisboa, Avenida Rovisco Pais, 1049-001, Lisboa, Portugal \\ ${ }^{\mathrm{c}}$ IBB, Institute for Bioengineering and Biosciences, Avenida Rovisco Pais, 1049-001, Lisboa, Portugal \\ ${ }^{\mathrm{d}}$ GreenCoLab - Green Ocean Technologies and Products Collaborative Laboratory, CCMAR, Algarve University, Portugal
}

\section{A R T I C L E I N F O}

\section{Keywords:}

Rhodosporidium toruloides

Tetradesmus obliquus

Pure and mixed cultures

Primary brewery wastewater

Lipids

\begin{abstract}
A B S T R A C T
Rhodosporidium toruloides and Tetradesmus obliquus pure and mixed cultures were grown on primary brewery wastewater (PBWW), with and without supplementation of sugarcane molasses (SCM) and urea. R. toruloides developed in pure cultures was not able to grow on PBWW, with or without supplementation, but grew in mixed cultures with the microalga $T$. obliquus in all media. In contrast, all T. obliquus pure cultures developed on PBWW, with and without supplementation, were able to develop. Higher biomass productivity (149.3 $\mathrm{mg} \mathrm{L}^{-1} \mathrm{~h}^{-1}$ ) was obtained for the mixed culture using PBWW supplemented with $10 \mathrm{~g} \mathrm{~L}^{-1}$ of SCM and $2 \mathrm{~g} \mathrm{~L}^{-1}$ of urea. Therefore, a strategy to stimulate lipid production by $R$. toruloides and T. obliquus grown in mixed cultures with higher SCM concentrations (20, 40 and $100 \mathrm{~g} \mathrm{~L}^{-1}$ of SCM) was developed. The maximum lipid content $(26.3 \%$ (w/w DCW)) was obtained for the mixed culture developed on PBWW, supplemented with $100 \mathrm{~g} \mathrm{~L}^{-1}$ of SCM plus $2 \mathrm{~g} \mathrm{~L}^{-1}$ of urea, at $\mathrm{t}=121 \mathrm{~h}$. This work demonstrated the feasibility of using PBWW supplemented with SCM and urea as culture medium to grow $R$. toruloides and T. obliquus and to produce microbial lipids.
\end{abstract}

\section{Introduction}

The fast-growing industrialization all over the world has led to an excessive increase in the pollution level, leading to global environmental hazards such as ozone depletion, global warming, acid rain, and contamination of water resources [1,2]. Therefore, it is necessary to develop efficient strategies to reduce the environmental impact of the waste produced by the different industries $[3,4]$. One of the major impacts of the rapid industrialization is the increase of water pollution, beyond the water scarcity, which strongly affects the availability and quality of water resources worldwide, threatening human health.

The brewery industry is an important economic segment of many countries, being beer the fifth most consumed beverage in the world [5, 6]. Due to the high consumption of beer worldwide, the amount of brewery wastewater that is produced is massive $[6,7]$.

To be discharge into the environment, the brewery wastewater should be previously treated to accomplish the environmental legislation of each country. Conventional primary brewery wastewater (PBWW) treatment intent to remove the largest particles that can cause operational problems during the BWW treatment and remove the suspended solids and organic matter content in the raw wastewater. The secondary brewery wastewater (SBWW) treatment aims to remove the biodegradable organic matter (dissolved or in suspension) and the remaining suspended solids $[8,9]$.

Oleaginous microorganisms such as yeasts and microalgae are able to accumulate intracellularly triacylglycerols (TAGs) as storage materials, which can reach more than $20 \%$ of the biomass weight, being considered a potential source of oil for the production of advanced biofuels (including biodiesel) [10] since the intracellular oil can be extracted or directly (in situ) converted into fatty acids alkyl esters (biodiesel) through a transesterification reaction [11]. Moreover, microbial growth does not need large arable land areas intended for food production, and do not have high water quality and fertilization requirements, which are advantages comparing to vegetable crops [12]. However, in spite of the

\footnotetext{
Abbreviations: PBWW, Primary Brewery Wastewater; SBWW, Secondary Brewery Wastewater; SCM, Sugarcane Molasses.

* Corresponding author.

E-mail address: teresa.lopessilva@lneg.pt (T. Lopes da Silva).
} 